\title{
Promotion of Biogasification Efficiency by Pretreatment and Bioaugmentation of Corn Straw with Microbial Consortium
}

\author{
Qili Zhu ${ }^{1,2+}$, Yanwei Wang ${ }^{1+}$, Furong Tan ${ }^{1}$, Bo $\mathrm{Wu}^{1}$, Lichun Dai ${ }^{1}$, Mingxiong $\mathrm{He}^{1 *}$, and Guoquan $\mathrm{Hu}^{1 *}$ \\ ${ }^{1}$ Biomass Energy Technology Research Center, Biogas Institute of Ministry of Agriculture, Chengdu, P. R. China, 610041 \\ ${ }^{2}$ National Agricultural Science and Technology Center, Chengdu, P. R. China, 610041
}

\begin{abstract}
To better understand the comparative effects between pretreatment and bioaugmentation methods on the promotion of corn straw biogasification efficiency, we analysed the cellulase activity, cellulose degradation rate, surface structure characteristics, and biogas production of corn straw that had been pretreated with aerobic microbial consortium (AMC). In addition, we also studied the effect of bioaugmentation using anaerobic microbial consortium (ANMC) on corn straw biogasification efficiency. The results from our study demonstrated that the cumulative methane generated from AMC and ANMC were $233.09 \mathrm{~mL} \cdot \mathrm{g}-1 \mathrm{VS}$ and $242.56 \mathrm{~mL} \cdot \mathrm{g}-1 \mathrm{VS}$, which was increased compared to the control by $6.89 \%$ and $11.23 \%$, respectively. We also observed that ANMC could also function to dramatically promote methane content during the anaerobic digestion of corn straw. This study demonstrated that AMC and ANMC were both able to promote the biogasification efficiency of corn straw, however, ANMC was found to perform better compared to AMC.
\end{abstract}

\section{Introduction}

The energy crisis and environmental pollution have become two major problems in the development of the world today (Yan et al., 2012). Straw biomass biogasification technology is one of the key technologies existing today that have the potential to effectively alleviate these problems. Indeed, straw biomass biogasification is widely employed for the treatment of organic waste and the production of methane (Bond and Templeton, 2011; Rouches et al., 2016). However, the efficiency of straw biogasification is currently relatively low due to its recalcitrance that has been demonstrated to restrict the resource utilization process of agricultural waste (Zheng et al., 2014). Therefore, some measurements like pretreatment and/or bioaugmentation should be adopted to increase biogas production from lignocellulosic material, further to resolve such technological difficulties.

Pretreatment technology can be carried out in an effort to improve lignocellulose hydrolysis and further promote methane yields (Hua et al, 2016). Previous studies have studied the effect of different pretreatment methods on the enhancement of the anaerobic digestion efficiency of lignocellulosic material. For example, methane production from reed which was pretreated with steam explosion was found to be increased by $89 \%$ (Lizasoain et al., 2016), while that of rice straw that was pretreated with fungus was observed to be increased by $78.3 \%$ (Mustafa et al., 2016). In general, the pretreatment methods utilized in biogas production can be classified into three different categories: physical, chemical, and biological (Wen et al., 2015). Biological pretreatment uses microorganisms to digest the cell walls of plant biomass and has generated attention within the field due to its low energy consumption, cost-effectiveness, and environmental friendliness (Agbor et al., 2011; Zheng et al., 2014). Microbial consortium represents one of the efficient biological pretreatment methods and has been demonstrated to be able to impose synergistic effects among different functional microorganisms. In addition, it can remove feedback inhibition of metabolites and promoting lignocellulose degradation efficiency in comparison with that when a single strain is used, or physical and chemical methods are used (Chandel and Singh, 2011; Zhong et al., 2016). The use of microbial consortium for pretreatment has been demonstrated to be advantageous for large-scale biomass production due to the fact that, in the majority of cases, lignocellulosic feedstock sterilization is not necessary, which could help to lower costs and save time (Bruni et al., 2010; Lu et al., 2009; Zheng et al., 2014; Hua et al, 2016). In general, there are two main microbial consortiums that are utilized for the pretreatment of lignocellulosic biomass. These include one type that gains directly from a special environment, such as rumen fluid or digested sludge. The other types, including MC1 (Hua et al, 2016; Yuan et al., 2016), WSD-5 (Wen et al., 2012), XDC-2 (Zhang et al., 2016), and MCHCA (Poszytek et al., 2016) are all generated from the natural environment (soil, sludge, etc.). It has been demonstrated that both two types of microbial consortium can function to promote the biogasification efficiency of straw inordinately.

Bioaugmentation, which is used to improve refractory organics catabolism via the addition of selected strain/s or mixed cultures to biological systems, was initially

\footnotetext{
* Corresponding author: hemingxiong@ caas.cn \& huguoquan@caas.cn.

+ Qili Zhu and Yanwei Wang contributed equally to this work.
} 
introduced into $\mathrm{AD}$ processes in recent years as an alternative method aimed to increase biofuel product yield, such as ethanol, hydrogen, and methane (Wei, 2016). In comparison with biological pretreatment methods aimed to improve biogas production from lignocellulosic materials, the bioaugmentation technique possesses certain advantages, including the requirement of less time, lower costs, less dry matter loss, and toxicity delimiting (Wei, 2016; Town and Dumonceaux, 2016). According to recent studies, the bioaugmentation technique was confirmed to accelerate acidification, further improving biogas production from lignocellulosic materials (Yang et al., 2016; Weiss et al., 2016).

The effects of pretreatment using different microbial consortium on the biogasification efficiency of lignocellulosic materials was studied previously (Wen et al., 2015), as well as the effects of different bioaugmentation patterns on biogas production (MartinRyals et al., 2015; Yang et al., 2016). However, there exist few studies regarding the comparative effects of pretreatment with either microbial consortium or bioaugmentation using lignocellulolytic microbes on the production of biogas from lignocellulosic biomass. In this study, we aim to understand which method performs better. The results obtained from this study are beneficial as they can be used to screen and optimize treatment methods for the efficient degradation of lignocellulose. In addition, these studies will provide further insight into resolving the technical bottleneck of the straw biogas project in China.

\section{Materials and methods}

\subsection{Experiment materials}

Corn straw was collected from farmland in a suburb of Chengdu, China. The collected straw was air-dried and shredded into $5 \mathrm{~mm}$ pieces. Digested sludge obtained from a pig farm was used as inoculum. Feedstock and inoculum characteristics are presented in Table 1.

Table 1. Characteristics of corn straw and digested sludge

\begin{tabular}{ccccc}
\hline & TS (\%) & VS (\%) & C (\%) & N (\%) \\
\hline $\begin{array}{c}\text { Corn } \\
\text { straw }\end{array}$ & $87.91 \pm 0.11$ & $78.81 \pm 0.04$ & $36.59 \pm 0.04$ & $0.73 \pm 0.02$ \\
$\begin{array}{c}\text { Digested } \\
\text { sludge }\end{array}$ & $2.92 \pm 0.05$ & $1.68 \pm 0.05$ & N.D. & N.D. \\
\hline
\end{tabular}

Note: N.D. None determination

Aerobic microbial consortium for pretreatment (AMC) : Constructed with the experiment including the Congo red screening, cellulase activity, and antagonism determination in sequence. It was comprised of Paenibacillus cucumis (1 strain, derived from bamboo insect), Bacillus altitudinis (2 strains, derived from rumen fluid), Bacillus subtilis (3 strains, derived from soil), and Lysinibacillus halotolerans (1 strain, derived from rumen fluid).

Anaerobic microbial consortium for bioaugmentation (ANMC): Stable, derived from bamboo insect, and enriched to 21 generations via successive generations.
Clostridum sp. was the dominate microorganism in ANMC.

Culture medium: $\mathrm{KH}_{2} \mathrm{PO}_{4} 1.0 \mathrm{~g}, \mathrm{NaCl} 0.1 \mathrm{~g}$, $\mathrm{MgSO}_{4} \bullet 7 \mathrm{H}_{2} \mathrm{O} 0.3 \mathrm{~g}, \mathrm{NaNO}_{3} 2.5 \mathrm{~g}, \mathrm{FeCl}_{3} 0.01 \mathrm{~g}, \mathrm{CaCl}_{2} 0.1$ $\mathrm{g}, \mathrm{H}_{2} \mathrm{O} 1 \mathrm{~L}$, and $0.5 \mathrm{~g} \cdot \mathrm{L}^{-1}$ cellulosic materials.

\subsection{Pretreatment of corn straw}

$5 \mathrm{~g}$ corn straw and $10 \mathrm{~mL}$ AMC liquid (10\% inoculation) were mixed with $100 \mathrm{~mL}$ medium in a $250 \mathrm{~mL}$ shake flask. The flask was then incubated at $30 \mathrm{C}$ with shaking at $160 \mathrm{rpm}$ for $3 \mathrm{~d}$. This was defined straw pretreatment solution, which can be used for following anaerobic digestion of AMC group directly. Additional samples were then dried and utilized for lignocellulose content determination and scanning electron microscope analysis.

\subsection{Anaerobic digestion}

Batch anaerobic digestion of pretreated corn straw was carried out as follows. The ratio of straw to digested sludge used was 1: 1 based on volatile solid content. A $500 \mathrm{~mL}$ glass bottle with a working volume of $350 \mathrm{~mL}$ was utilized for this assay. A total of 3 test groups were set up as follows.

AMC: Straw pretreatment solution (5 g corn straw, $100 \mathrm{~mL}$ medium and $10 \mathrm{~mL}$ aerobic microbial consortium) was combined with $235 \mathrm{~g}$ digested sludge after $3 \mathrm{~d}$ pretreatment.

ANMC: $5 \mathrm{~g}$ corn straw, $100 \mathrm{~mL}$ medium and $10 \mathrm{~mL}$ anaerobic microbial consortium were directly added to 235 g digested sludge;

Control group (Ctrl): 5 g corn straw, $100 \mathrm{~mL}$ medium and $10 \mathrm{~mL}$ ultrapure water were added to $235 \mathrm{~g}$ digested sludge.

In addition, a blank control group (235 g digested sludge only) was also used. All reactions were carried out under mesophilic $(35 \pm 2) \quad \mathrm{C}$ conditions using a water bath. A total of three replicates were carried out for this assay.

\subsection{Analysis methods}

\subsubsection{Conventional physical and chemical indicators analysis}

Total solids (TS) and volatile solids (VS) were both measured according to standard methods (APHA, 2012), while lignocellulose contents were measured using standard methods described by NREL in the USA (National Renewable Energy Laboratory, 2015). Carbon and nitrogen were both measured using a Vario MICRO select elemental analyzer (Elementar, Mt. Laurel, NJ, USA).

Biogas production was analysed using water displacement, while biogas composition was quantified using gas chromatography equipment (GC122, Shanghai Instrument-electric Analysis Instrument Co., Ltd., Shanghai, China) equipped with a thermal conductivity detector (TCD) (Zhu, et al. 2017). The stainless-steel 
column that was used for these analyses was packed with Porapak Q. The injector, oven, and detector temperatures were set to 120,120 , and $150 \mathrm{C}$, respectively. Nitrogen was used as the carrier gas and the flow was maintained at $30 \mathrm{~mL} \cdot \mathrm{min}-1$.

The concentration of volatile fatty acids was analysed using a gas chromatography instrument (GC102, Shanghai Instrument-electric Analysis Instrument Co., Ltd., Shanghai, China) equipped with a flame ionization detector (FID). The injector, oven, and detector temperatures were set to 160,210 , and $230 \mathrm{C}$, respectively. Nitrogen was used as the carrier gas, and the flow rate was maintained at $30 \mathrm{~mL} \cdot \mathrm{min}-1$.

\subsubsection{Enzyme activity analysis}

Single strain and microbial consortium were cultured in $\mathrm{HM}$ medium (KH2PO4 $1.0 \mathrm{~g}, \mathrm{NaCl} 0.1 \mathrm{~g}, \mathrm{MgSO} 4 \cdot 7 \mathrm{H} 2 \mathrm{O}$ $0.3 \mathrm{~g}, \mathrm{NaNO} 32.5 \mathrm{~g}, \mathrm{FeCl} 30.01 \mathrm{~g}, \mathrm{CaCl} 20.1 \mathrm{~g}$, CMC-Na $5 \mathrm{~g}$, and $\mathrm{H} 2 \mathrm{O} 1 \mathrm{~L}$ ) at $30 \mathrm{C}$ for $3 \mathrm{~d}$. Liquid samples were centrifuged at $8,000 \mathrm{rpm}$ for $10 \mathrm{~min}$. This centrifuged sample was used as crude enzyme liquid, and its activity was measured according to standard methods (DNS) (Oppert et al., 2010; Shi et al., 2011). Carboxymethyl cellulose enzyme activity (CMCase), cellobiase, and filter paper enzyme (FPase) were also analyzed in this study. The filter paper (Whatman $1,1 \mathrm{~cm} \times 6 \mathrm{~cm}$ ), sodium carboxymethylcellulose ( $1 \%)$, and salicin $(2 \%)$ were used as substrates for FPase, CMCase and cellobiase analysis, respectively. The enzyme activity was determined according to the reducing sugar (glucose) concentration that was generated from the enzyme catalysis of enzyme protein in unit time (Shi et al., 2011).

\subsubsection{Scanning electron microscope analysis}

Straw samples prior to and following pretreatment were utilized for scanning electron microscopy (HITACHI TM-1000, Japan) analysis.

\section{$2.516 S$ rDNA analysis}

Sludge samples were collected from both treated and untreated digesters following a 40-d anaerobic digestion. These samples were stored in an ultra-low temperature freezer at $-80 \quad \mathrm{C}$ for future DNA extraction and subsequent microbial community structure analysis. These analyses were completed by a Sequencing company.

\section{Results and discussion}

\subsection{Lignocellulose degradation rate of straw}

The lignocellulose degradation rate is one indicator which represents the biodegradability of straw biomass following pretreatment. Both the lignocellulose contents and the degradation rate of corn straw pretreated with AMC are shown in Table 2. The cellulose, hemicellulose, and lignin content of corn straw that was pretreated for 3 $\mathrm{d}$ using AMC were $0.25,0.23$ and $0.14 \mathrm{~g} \cdot \mathrm{g}-1$, respectively. These levels were comparatively reduced compared to the control by $16.7 \%, 17.9 \%$, and $12.5 \%$, respectively. These results demonstrated that $\mathrm{AMC}$ can affect degrading cellulose. According to these results, we found that hemicellulose was degraded faster in comparison to cellulose. This was due to the fact that the primary composition of hemicellulose was carbohydrates, which are easy to degrade. These included xylan, glucuronoxylan, arabinoxylan, glucomannan, and xyloglucan, which have increased variability in structure and composition compared to cellulose, and function to maintain a comparatively higher degradation rate during the pretreatment process (Van Dyk and Pletschke, 2012). Therefore, in contrast to cellulose, hemicellulose was shown to be relatively easy to hydrolyze, with the monomeric sugars and acetic acid produced able to be subjected to bioconversion for the production of biogas and other useful byproducts (Nanda et al., 2014). Cellulose is a polymer that consists of glucose units connected by $\beta-1-4$ glycosidic bonds (Li et al., 2014) and is wrapped by lignin, making it more difficult to hydrolyze. Lignin is a complex polyphenyl aromatic compound that linked via ester bonds. It tightly binds cellulose and hemicellulose to form plant primary and secondary cell walls (Nanda et al., 2014). In addition, lignin is resistant to degradation and acts as an obstacle for the effective utilization of cellulose and hemicellulose (van Kuijk et al., 2015). Therefore, the aim of microbial consortium pretreatment was to destroy the lignocellulosic structure, which can assist in improving the hydrolysis process, further to promote the biogasification efficiency of lignocellulosic materials.

Table 2. Lignocellulose contents of corn straw before and after pretreatment by AMC

\begin{tabular}{cccc}
\hline & $\begin{array}{c}\text { Before } \\
\text { pretreatment } \\
\left(\mathrm{g} \cdot \mathrm{g}^{-1}\right)\end{array}$ & $\begin{array}{c}\text { After } \\
\text { pretreatment } \\
/\left(\mathrm{g} \cdot \mathrm{g}^{-1}\right)\end{array}$ & $\begin{array}{c}\text { Degradation } \\
\text { rate }(\%)\end{array}$ \\
\hline Cellulose & $0.30 \pm 0.80$ & $0.25 \pm 0.95$ & 16.7 \\
Hemicellulose & $0.28 \pm 0.25$ & $0.23 \pm 0.29$ & 17.9 \\
Lignin & $0.16 \pm 0.26$ & $0.14 \pm 0.03$ & 12.5 \\
\hline
\end{tabular}

\subsection{Cellulase activity}

Cellulose degradation is a complex biological process and is accomplished with the synergistic effects of various cellulases. Therefore, we analyzed the activity of three common cellulases, including FPase, CMCase, and Cellobiase (Xu et al., 2018) in this study. As depicted in Table 3, the FPase, CMCase, and Cellobiase activity levels were determined to be $0.33 \mathrm{U} \cdot \mathrm{mL}-1,1.69 \mathrm{U} \cdot \mathrm{mL}-1$ and $0.013 \mathrm{U} \cdot \mathrm{mL}-1$, respectively, all of which were beyond the superior limit of a single strain. This result agrees with the conclusion that lignocellulose degradability of microbial consortium is much greater than that of a single strain (Wang et al., 2011a). 
Table 3. Cellulase activity of single strain and microbial consortium AMC

\begin{tabular}{ccc}
\hline & $\begin{array}{c}\text { Single strain } \\
\left(\mathrm{U} \cdot \mathrm{mL}^{-1}\right)\end{array}$ & $\mathrm{AMC}\left(\mathrm{U} \cdot \mathrm{mL}^{-1}\right)$ \\
\hline FPase & $0.05 \sim 0.17$ & 0.33 \\
CMCase & $0.3 \sim 0.8$ & 1.69 \\
Cellobiase & $0 \sim 0.01$ & 0.013 \\
\hline
\end{tabular}

\subsection{Scanning electron microscope analysis}

The apparent morphological structural changes of corn straw prior to and following microbial consortium AMC pretreatment are depicted in Fig. 1. Obvious structural destruction was observed, which was accompanied by a larger specific surface area in pretreated straw. The untreated straw surface was observed to be regular and smooth (Fig. 1a), while the pretreated straw surface was observed to be rough and fragmented (Fig. 1b). This was due to the fact that various cellulases which were secreted from microbial consortium AMC exerted synergistic effects on the destruction of the lignocellulosic structure, which benefited the efficient degradation of lignocellulose and its subsequent hydrolysis and fermentation (Zhong et al., 2016).
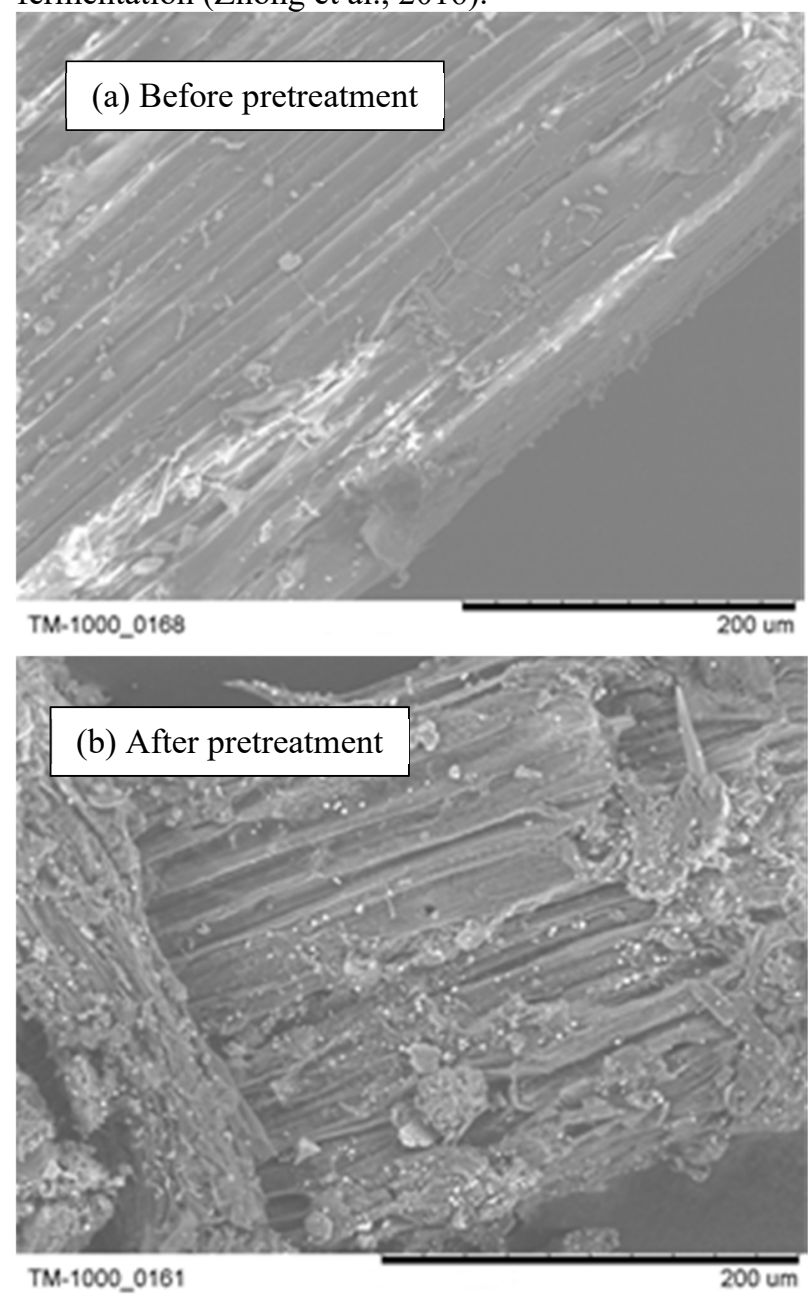

Fig.1 Electric microscope scanning graph of untreated and pretreated corn straw $(\times 500$ times $)$

\subsection{Performance of anaerobic digestion}

Biomethane production is a key indicator for the evaluation of biomass energy conversion and biogasification efficiency ( $\mathrm{Hu}$ et al., 2015). We studied the cumulative amount of methane generated from untreated and pretreated corn straw (Fig. 2) in order to understand the differences between the effect of pretreatment with AMC versus bioaugmentation with ANMC on corn straw biogas production. According to the results from this study, the cumulative amounts of methane that was generated from ANMC and AMC were $242.56 \mathrm{~mL} \cdot \mathrm{g}-1 \mathrm{VS}$ and $233.09 \mathrm{~mL} \cdot \mathrm{g}-1 \mathrm{VS}$, which was increased with respect to the control by $11.23 \%$ and $6.89 \%$, respectively. In general, ANMC was observed to perform better than AMC. This could be due to the fact that AMC pretreatment promoted the process of straw hydrolysis to saccharification, but meanwhile consumed some carbohydrates in order to meet the demands of its growth. Therefore, there were less carbohydrates remaining to be used for subsequent anaerobic digestion, further offsetting the promoting effect of $\mathrm{AMC}$ on the biogasification efficiency of straw. In contrast, ANMC primarily consists of Clostridum sp., a species that enhances bacterial and archaeal diversity and quantities, and further promotes the hydrolysis and biogas production of lignocellulosic materials (Aydin, 2016). Therefore, when the previous advantages of bioaugmentation over pretreatment methods are taken into consideration, we found that bioaugmentation with ANMC was better choice for promoting biomethane production of corn straw, compared with pretreatment using AMC.

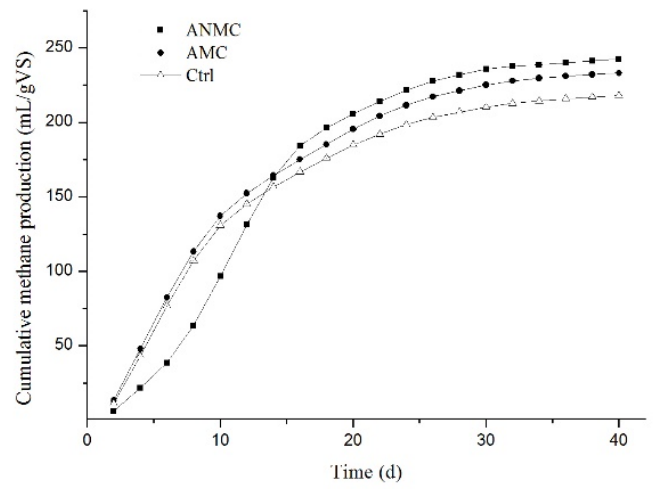

Fig.2 Changes of cumulative methane production during anaerobic digestion of corn straw

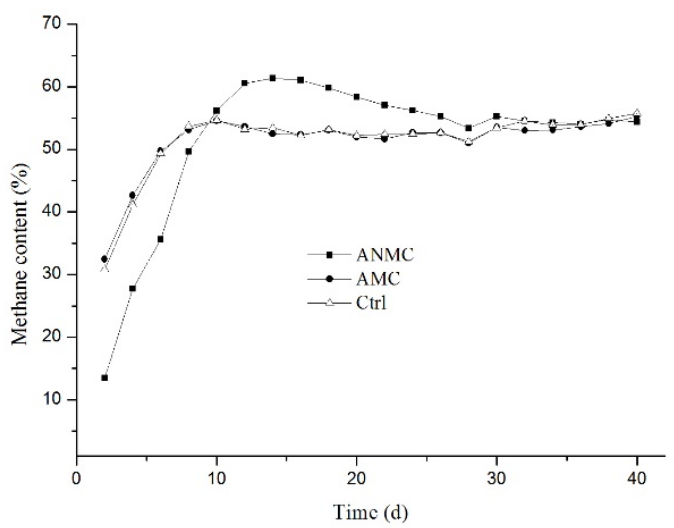

Fig.3 Changes of methane content during anaerobic digestion of corn straw 
In this study, we also analysed methane content (Fig. 3). Changes in methane content of AMC were found to be similar to control samples, which were found to gradually increase to approximately $50 \%$, where they remained stable following $10 \mathrm{~d}$. In regard to ANMC, we determined that its methane content was less than that of AMC and control samples at the initial stage, but exceeded control levels up to $61.4 \%$ following $10 \mathrm{~d}$, followed by a gradual decrease where it remained stable at approximately 54\%. These results demonstrated that bioaugmenting ANMC could function to improve corn straw methane content during the anaerobic digestion process.

Volatile fatty acids (VFAs) represent important indicators for the evaluation of the balance of both hydrolytic acidification and methane production (Wang et al., 2009). VFAs (acetic acid, propionic acid, and butyrate acid) generated from both untreated and pretreated corn straw are depicted in Fig. 4. Throughout the process, from the start to $16 \mathrm{~d}$, the VFAs concentration of ANMC was observed to be remarkably higher compared to that of AMC and control samples. This is likely due to the fact that Clostridum sp. from ANMC resulted in the production of short chain volatile substances during the metabolic process. The peak value was observed to appear at $6 \mathrm{~d}$, which was increased to $2169.4 \mathrm{mg} \cdot \mathrm{L}-1$. It has been previously demonstrated that VFAs have only a little effect on anaerobic digestion when it is at a concentration of less than $4125 \mathrm{mg} \cdot \mathrm{L}-1$ (Wang et al., 2011b). In addition, these were found to produce short chain volatile acids, including acetic acid and butyric acid, which were beneficial for the subsequent methanogenesis process (Yuan et al., 2016). That explains why the cumulative methane production of ANMC was observed to be the greatest among the three test groups, even though its VFA concentration was still the highest.

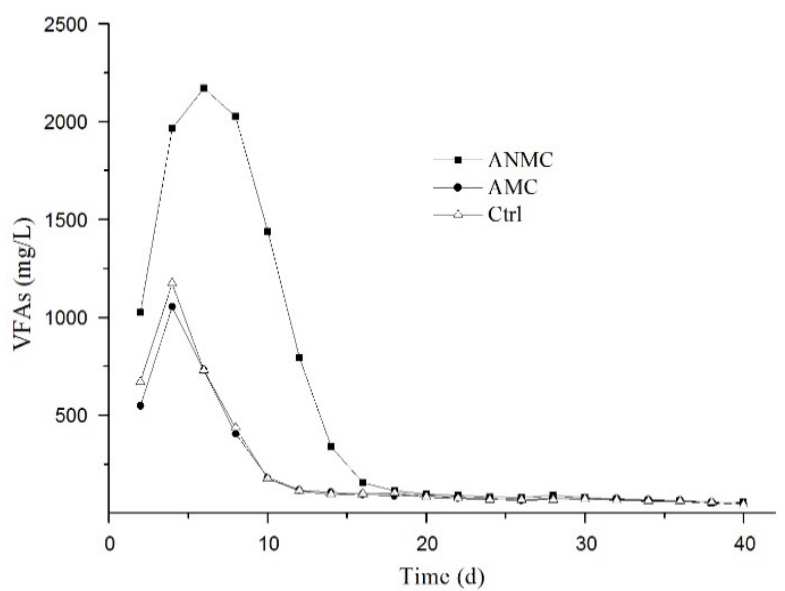

Fig.4 Changes of VFAs concentration during anaerobic digestion of corn straw

In general, both pretreatment with $\mathrm{AMC}$ and bioaugmentation with ANMC were found to have a promoting effect on the biogasification efficiency of corn straw, however, these effects were still limited in comparison to other related studies (Zhong et al., 2011; Zhang et al., 2015). For example, the total biogas and methane production generated from straw that had been pretreated with microbial consortium for $15 \mathrm{~d}$ was found to be increased by $33.1 \%$ and $75.6 \%$, respectively (Zhong et al., 2011). According to the study by Zhang et al., bioaugmentation using Acetobacteroides hydrogenigenes was found to increase cellulose and hemicellulose removal rates and improve methane yield by $19-23 \%$ throughout the anaerobic digestion process of corn straw (Zhang et al., 2015). However, no matter whether pretreatment or bioaugmentation was utilized, the total biogas and methane production in this study was found to increase only by $13.02 \%$ and $11.23 \%$. This could be attributed to the pretreatment time used in this study (only 3 days), which failed to efficiently degrade lignocellulosic materials into soluble substrate, compared with other studies used much longer times (Hua et al., 2016; Yuan et al., 2016; Zhong et al., 2011). An alternative reason could be related to the fact that the parameters used for this test were not optimized, which could have inhibited the efficient operation of anaerobic digestion. In addition, these results also indicated that the promoting effect of microbial consortium that was generated in this study was limited and requires further optimization. Fortunately, the comparative results regarding the effects of pretreatment with microbial consortium and bioaugmenting the lignocellulolytic microbes on the biogas production of lignocellulosic biomass could provide novel insights related to the screening and optimization of an efficient treatment method for the degradation of lignocellulose.

\subsection{Microbiological Community structure analysis}

We studied the microbiological community structure to understand differences in the type and quantity of microorganisms that exist between pretreatment and bioaugmentation methods (Fig. 5 and Fig. 6). We found that with both bacterium and archaeas, the microbial community compositions at the phylum level were similar in AMC and ANMC. In general, bacterium was found to be primarily comprised of Firmicutes and Bacteroidetes, while Euryarchaeota and Crenarchaeota were mainly archaeas. The phylum of Firmicute comprises numerous hydrolytic and acidogenic bacterium, facilitating the transformation of biopolymers to organic acids (Stolze et al. 2015). Importantly, methanosarcina was found to be the dominant microorganism in both AMC and ANMC, which suggests that it plays a leading role in the production of biogas from corn straw treated with AMC and ANMC. We found that methanosarcina accounted for $8 \%$ in ANMC, while only $7 \%$ in AMC. These results agree with previous results showing that ANMC performed better than $\mathrm{AMC}$ regarding the enhancement of biogas production.

It is important to note that we observed an altered microbiological community structure in ANMC. Therefore, this suggests that the introduced strains were undetectable in the microbial community at the completion of anaerobic digestion, as similarly described by Cater et al. (Cater et al., 2015). The observed changes in the microbial community could be due to competition for substrate and/or specific ecological niches between bioaugmented microorganism and indigenous 
populations, or due to inhibition resulting from antibiotics or some type of metabolic inhibitor (Veen et al., 1997).

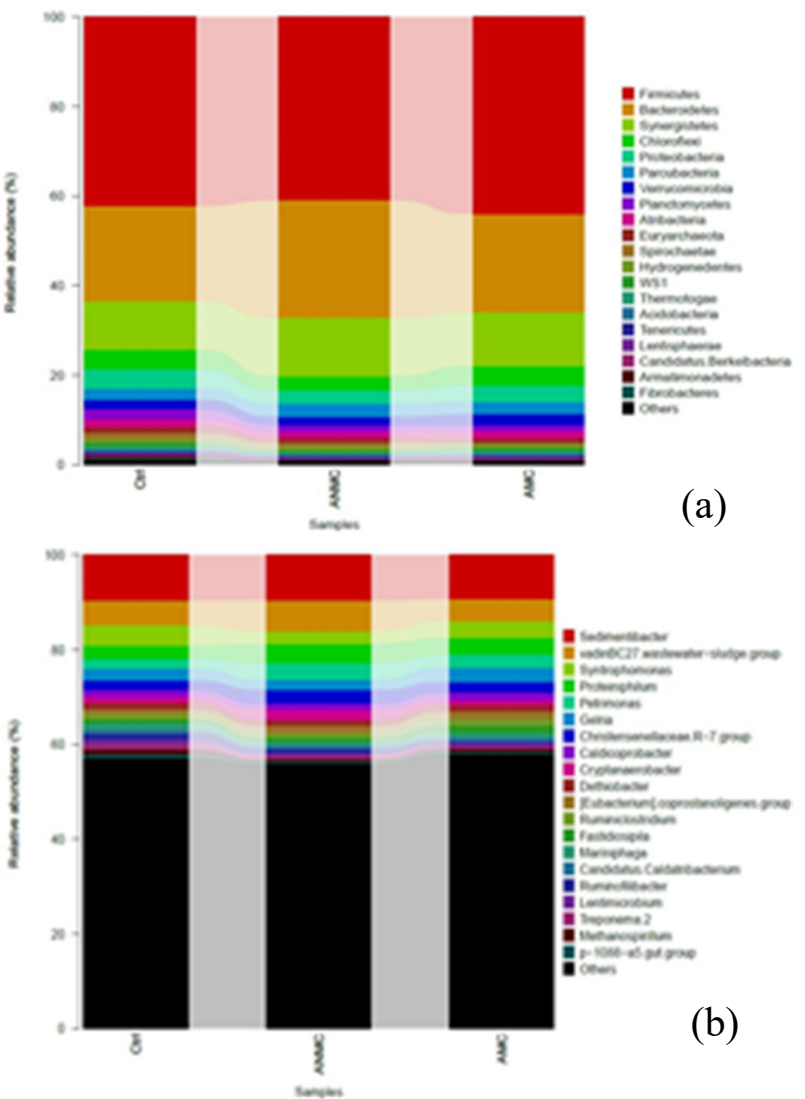

Fig.5 Relative abundance of bacteria at (a) phylum leveland (b) genus level

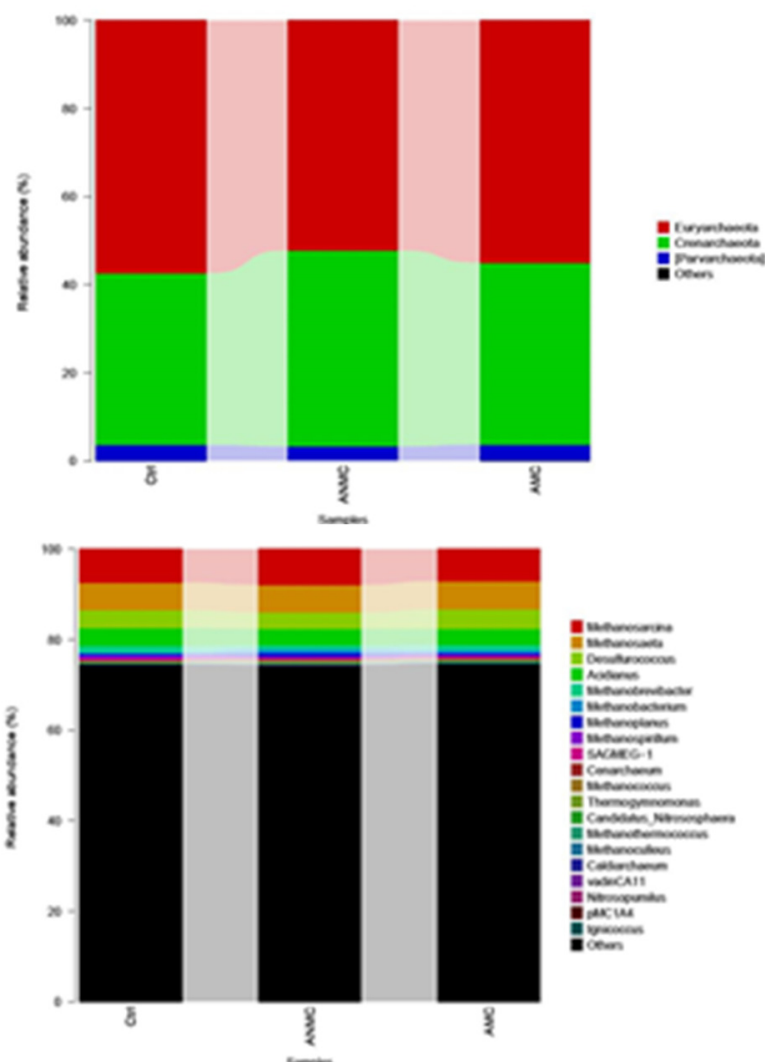

Fig.6 Relative abundance of archea at (a) phylum level and (b) genus level

\section{CONCLUSIONS}

In this study, we examined the comparative effects of pretreatment and bioaugmentation methods on biogas production of lignocellulosic biomass. We demonstrate that the cumulative methane generated from corn straw which was pretreated with microbial consortium (AMC) and bioaugmentated with anaerobic lignocellulolytic microbes (ANMC) was increased by $6.89 \%$ and $11.23 \%$, respectively. ANMC was observed to perform better compared to AMC in regards to its ability to improve biogas production. In particular, we found that ANMC was able to dramatically promote methane content throughout the anaerobic digestion of corn straw. When taking into consideration the previously described advantages of bioaugmentation over pretreatment methods, ANMC was determined to be a better choice for the promotion of biogasification efficiency.

\section{Acknowledgements}

This work was financially supported by the Key Research and Development Project of Science and Technology Department of Sichuan Province (No. 2018SZ0283), Local financial funds of National Agricultural Science and Technology Center (No. NASC2019TI06), National Natural Science Foundation of China (No. 31600012) and Sichuan Science and Technology Program (No. 2018JY0216).

\section{References}

1. V.B. Agbor, N. Cicek, R. Sparling, A. Berlin, D.B. Levin. Biomass pretreatment: fundamentals toward application. Biotechnol. Adv. 29 : 675-685 (2011)

2. APHA (American Public Health Association). Standard Methods for the Examination of Water and Wastewater, Washington DC, USA (2012)

3. S. Aydin. Enhancement of microbial diversity and methane yield by bacterial bioaugmentation through the anaerobic digestion of Haematococcus pluvialis. Appl. Microbiol. Biotechnol. 100 : 5631-5637 (2016)

4. T. Bond, M.R. Templeton. History and future of domestic biogas plants in the developing world. Energy Sustainable Dev. 15 : 347-354 (2011).

5. E. Bruni, A.P. Jensen, I. Angelidaki. Comparative study of mechanical, hydrothermal, chemical and enzymatic treatments of digested biofibers to improve biogas production. Bioresour. Technol. 101: 8713-8717 (2010)

6. M. Cater, L. Fanedl, S. Malovrh, R.M. Logar. Biogas production from brewery spent grain enhanced by bioaugmentation with hydrolytic anaerobic bacteria. Bioresour. Technol. 186 : 261-269 (2015)

7. A.K. Chandel, O.V. Singh. Weedy lignocellulosic feedstock and microbial metabolic engineering: advancing the generation of 'Biofuel'. Appl. Microbiol. Biotechnol. 89(5): 1289-1303 (2011)

8. Y. Hu, Y.Z. Pang, H.R. Yuan, D.X. Zou, Y.P. Liu, B.N. Zhu, M.J. Chufo, X.J. Li. Promoting anaerobic 
biogasification of corn stover through biological pretreatment by liquid fraction of digestate (LFD). Bioresour. Technol. 175: 167-173 (2015)

9. B.B. Hua, J.L. Dai, B. Liu, H. Zhang, X.F. Yuan, X.F. Wang, Z.J. Cui. Pretreatment of non-sterile, rotted silage maize straw by the microbial community $\mathrm{MC1}$ increases biogas production. Bioresour. Technol. 216: 699-705 (2016)

10. Q.X. Li, W.T. Ng, J.C. Wu. Isolation, characterization and application of a cellulosedegrading strain Neurospora crassa S1 from oil palm empty fruit bunch. Microb. Cell. Fact. 13: 157-165 (2014)

11. J. Lizasoain, M. Rincon, F. Theuretzbacher, R. Enguidanos, P.J. Nielsen, A. Potthast, T. Zweckmair, A. Gronauer, A. Bauer. Biogas production from reed biomass: Effect of pretreatment using different steam explosion conditions. Biomass Bioenerg. 95: 84-91 (2016)

12. A. Martin-Ryals, L. Schideman, P. Li, H. Wilkinson, R. Wagner. Improving anaerobic digestion of a cellulosic waste via routine bioaugmentation with cellulolytic microorganisms. Bioresour. Technol. 189, 62-70 (2015)

13. A.M. Mustafa, T.G. Poulsen, K.C. Sheng. Fungal pretreatment of rice straw with Pleurotus ostreatus and Trichoderma reesei to enhance methane production under solid-state anaerobic digestion. Appl. Energy 180: 661-671 (2016)

14. S. Nanda, J. Mohammad, S.N. Reddy, J.A. Kozinski, A.K. Dalai. Pathways of lignocellulosic biomass conversion to renewable fuels. Biomass Convers. Biorefin. 4: 157-191 (2014)

15. National Renewable Energy Laboratory, Inc. Standard procedures for biomass compositional analysis [EB/OL]. [2015-7-11]. http://www.nrel.gov/biomass/analytical_procedures. html.

16. C. Oppert, W.E. Klingeman, J.D. Willis, B. Oppert, J.L. Jurat-Fuentes. Prospecting for cellulolytic activity in insect digestive fluids. Comp. Biochem. Physiol. B-Biochem. Mol. Biol. 155: 145-154 (2010)

17. K. Poszytek, M. Clezkowska, A. Sklodowska, L. Drewniak. Microbial Consortium with High Cellulolytic Activity (MCHCA) for Enhanced Biogas Production. Front. Microbiol. 7: 1-11 (2016)

18. E. Rouches, I. Herpoël-Gimbert, J.P. Steyer, H. Carrere. Improvement of anaerobic degradation by white-rot fungi pretreatment of lignocellulosic biomass: A review. Renew. Sust. Energ. Rev. 59: 179-198 (2016)

19. W. Shi, S.Y. Ding, J.S. Yuan. Comparison of insect gut cellulase and xylanase activity across different insect species with distinct food sources. BioEnergy Res. 4(1): 1-10 (2011)

20. Y. Stolze, M. Zakrzewski, I. Maus, F. Eikmeyer, S. Jaenicke, N. Rottmann, C. Siebner, A. Pühler, A. Schlüter. Comparative metagenomics of biogas- producing microbial communities from productionscale biogas plants operating under wet or dry fermentation conditions. Biotechnol. Biofuels 8: 14 (2015)

21. J.R. Town, T.J. Dumonceaux. Laboratory-scale bioaugmentation relieves acetate accumulation and stimulates methane production in stalled anaerobic digesters. Appl. Microbiol. Biotechnol. 100: 10091017 (2016)

22. J.S. van Dyk, B.I. Pletschke. A review of lignocellulose bioconversion using enzymatic hydrolysis and synergistic cooperation between enzymes - factors affecting enzymes, conversion and synergy. Biotechnol. Adv. 30: 1458-1480 (2012)

23. S.J. van Kuijk, A.S. Sonnenberg, J.J. Baars, W.H. Hendriks, J.W. Cone. Fungal treated lignocellulosic biomass as ruminant feed ingredient: a review. Biotechnol. Adv. 33: 191-202 (2015)

24. J.A. Veen, L.S. Overbeek, J.D. Elsas. Fate and activity of microorganisms introduced into soil. Microbiol. Mol. Biol. Rev. 61: 121-135 (1997)

25. W.D. Wang, L. Yan, Cui, Z.J., Y.M. Gao, Y.J. Wang, R.Y. Jing. Characterization of a microbial consortium capable of degrading lignocellulose. Bioresour. Technol. 102(19): 9321-9324 (2011a)

26. X.J. Wang, G.H. Yang, Y.Z. Feng, G.X. Ren, X.H. Han, Z.L. Song. Anaerobic co-digestion effects of manure and straw and analysis of influencing factors. Agro-Environment Sci. 30(12): 2594-2601 (2011b)

27. Y.Y. Wang, Y.L. Zhang, J.B. Wang, L. Meng. Effects of volatile fatty acid concentrations on methane yield and methanogenic bacteria. Biomass Bioenerg. 33: 848-853 (2009)

28. S. Weiss, W. Somitsch, I. Klymiuk, S. Trajanoski, G.M. Guebitz. Comparison of biogas sludge and raw crop material as source of hydrolytic cultures for anaerobic digestion. Bioresour. Technol. 207: 244251 (2016)

29. Wei, S.Z. The application of biotechnology on the enhancing of biogas production from lignocellulosic waste. Appl. Microbiol. Biotechnol. 100(23): 98219836 (2016)

30. B.T. Wen, X.F. Yuan, Y.Z. Cao, Y. Liu, X.F. Wang, Z.J. Cui. Optimization of liquid fermentation of microbial consortium WSD-5 followed by saccharification and acidification of wheat straw. Bioresour. Technol. 118: 141-149 (2012)

31. B.T. Wen, X.F. Yuan, Q.X. Li, J.J. Liu, J.W. Ren. Wang, X.F., Cui, Z.J. Comparison and evaluation of concurrent saccharification and anaerobic digestion of Napier grass after pretreatment by three microbial consortia. Bioresour. Technol. 175: 102-111 (2015)

32. X.Q. Xu, M.M. Lin, Q. Zang, S. Shi. Solid state bioconversion of lignocellulosic residues by Inonotus obliquus for production of cellulolytic enzymes and saccharification. Bioresour. Technol. 247: 88-95 (2018) 
33. Z. Yang, R. Guo, X. Xu, L. Wang, M .Dai. Enhanced methane production via repeated batch bioaugmentation pattern of enriched microbial consortia. Bioresour. Technol. 216: 471-477 (2016)

34. L. Yan, Y.M. Gao, Y.J. Wang, Q. Liu, Z.Y. Sun, B.R. Fu, X. Wen, Z.J. Cui, W.D. Wang. Diversity of a mesophilic lignocellulolytic microbial consortium which is useful for enhancement of biogas production. Bioresour. Technol. 111: 49-54 (2012)

35. X.F. Yuan, L. Ma, B.T. Wen, D.Y. Zhou, M. Kuang, W.H. Yang, Z.J. Cui. Enhancing anaerobic digestion of cotton stalk by pretreatment with a microbial consortium (MC1). Bioresour. Technol. 207: 293301 (2016)

36. D.D. Zhang, Y. Wang, D. Zheng, P. Guo, W. Cheng, Z.J. Cui. New combination of xylanolytic bacteria isolated from the lignocellulose degradation microbial consortium XDC-2 with enhanced xylanase activity. Bioresour. Technol. 221: 686-690 (2016)

37. J. Zhang, R.B. Guo, Y.L. Qiu, J.T. Qiao, X.Z. Yuan, X.S. Shi, C.S. Wang. Bioaugmentation with an acetate-type fermentation bacterium Acetobacteroides hydrogenigenes improves methane production from corn straw. Bioresour Technol. 179: 306-313 (2015)

38. Y. Zheng, J. Zhao, F.Q. Xu, Y.B. Li. Pretreatment of lignocellulosic biomass for enhanced biogas production. Prog. Energy Combust. Sci. 42: 35-53 (2014)

39. C. Zhong, C.M. Wang, X.F. Wang, H.H. Jia, P. Wei, Y. Zhao. Enhanced biogas production from wheat straw with the application of synergistic microbial consortium pretreatment. RSC Adv. 6(65): 6018760195 (2016)

40. W.Z. Zhong, Z.Z. Zhang, Y.J. Luo, S.S. Sun, W. Qiao, M. Xiao. Effect of biological pretreatments in enhancing corn straw biogas production. Bioresour. Technol. 102: 11177-11182 (2011)

41. Q.L. Zhu, L.C. Dai , B. Wu, F.R. Tan, W.G. Wang, X.Y. Tang, Y.W. Wang, M.X. He, G.Q. Hu. Integrated methane and ethanol production from livestock manure and soybean straw. BioResources 12(2): 2284-2295 (2017) 